\title{
A New Generation Method for Spatial-Temporal Correlated MIMO Nakagami Fading Channel
}

\author{
Qiu-Ming Zhu, ${ }^{1}$ Xiang-Bin Yu, ${ }^{1}$ Jun-Bo Wang, ${ }^{1,2,3}$ Da-Zhuan Xu, ${ }^{1}$ and Xiao-Min Chen ${ }^{1}$ \\ ${ }^{1}$ College of Electronic Information Engineering, Nanjing University of Aeronautics and Astronautics, Nanjing 210016, China \\ ${ }^{2}$ School of Software, Tsinghua University, Beijing 100084, China \\ ${ }^{3}$ National Mobile Communications Research Laboratory, Southeast University, Nanjing 210008, China
}

Correspondence should be addressed to Qiu-Ming Zhu, zhuqiuming@nuaa.edu.cn

Received 10 July 2011; Accepted 5 September 2011

Academic Editor: Yan Zhang

Copyright (C) 2012 Qiu-Ming Zhu et al. This is an open access article distributed under the Creative Commons Attribution License, which permits unrestricted use, distribution, and reproduction in any medium, provided the original work is properly cited.

\begin{abstract}
A new generation method for spatial and temporal correlated multiple-input multiple-output (MIMO) Nakagami fading channel is proposed, which has low complexity and is applicable for arbitrary fading parameters and prespecified correlation coefficients of different subchannel. The new scheme can be divided into two steps: (1) generate independent Nakagami fading sequences for each subchannel based on a novel rejection method; (2) introduce the temporal and spatial correlation based on the relationships between Rayleigh, Gamma, and Nakagami random processes. The analysis and simulation results show that the proposed simulator has a good agreement with the theoretical model on fading envelope distribution, spatial-temporal correlation characteristic.
\end{abstract}

\section{Introduction}

In the past decades, more and more attentions have been given to MIMO system for its high channel capacity [1]. Many studies and measured data also showed channel fadings on different antennas are correlated. Moreover, the Nakagami fading encompasses Rayleigh and Rice fadings and shows good performance in matching experimental data with different fading parameter $m$ [2]. Hence, correlated MIMO channel modeling and capacity evaluation become the interest of many researchers recently $[3,4]$. However, few published works focus on simple and accurate generating of spatial-temporal correlated MIMO Nakagami fading channel which is important for design or laboratory test of MIMO systems.

A number of methods for generating independent Nakagami fading channels are available in the literature. Yip and Ng's method [5] based on the product of square-root beta random variates (RVs) and Rayleigh RVs is accurate but only valid for $m<1$. The decomposition method [6] is also only valid for integer or half-integer value of $m$. Beaulieu and Cheng's method [7] based on the inverse cumulative distribution function of Nakagami distribution is suitable for arbitrary $m$ but requires a different set of coefficients by numerical computation or curve fitting. The rejection method in $[8,9]$ is accurate and universal but needs a set of constant coefficients determined empirically or lacks efficiency. In light of this fact, a novel simple and highefficient generation method for arbitrary $m$ is proposed in our simulator which yields an excellent accuracy.

Yip and Ng's method and the decomposition method are also suitable for generating temporal correlated Nakagami fadings with specific values of $m$. Recently, Ma and Zhang [10] proposed a universal method by constructing a new map relation between Gaussian and Nakagami sequences, but it is quite difficult to calculate the autocorrelation of the corresponding input Gaussian sequence. In [11], a simple method called rank-matching was proposed to introduce prespecified temporal correlation by rearranging the independent Nakagami sequence, while the author addressed the relationship between Rayleigh and Nakagami envelope in an approximation manner. In $[6,12,13]$, techniques of channel correlation matrix decomposition for generating spatial correlated channels were reported.

In this paper, we propose a new framework for generating the correlated MIMO Nakagami fading channels which possess (1) arbitrary and different $m$ for each subchannel; (2) arbitrary and different average fading power; (3) arbitrarily 
prespecified temporal correlation coefficients; (4) arbitrary spatial correlation coefficient matrix. The new method consists of two steps: generate independent Nakagami sequence and introduce spatial-temporal correlation. Additionally, the simulation error is also analyzed and testified by numerical examples.

The rest of the paper is organized as follows. In Section 2, a general description of MIMO channel is given. In Section 3, the new framework for simulating the correlated Nakagami fading MIMO channel is described and analyzed. In Section 4, the generation approach is verified through numerical simulation. Finally, the conclusions are drawn in Section 5.

Throughout the paper, we use $[\cdot]^{T}$ to denote transposition. $E[\cdot]$ and $\operatorname{var}[\cdot]$ means the expectation and variance, respectively. $\rho_{R}^{k}(\tau)$ is the temporal correlation coefficient (TCC) of the $k$ th subchannel, and $\rho_{R}(k, 1)$ is the spatial correlation coefficient (SCC) between subchannel $k$ and $l$. $\Upsilon(a, b)$ denotes the Gamma distribution with distribution parameters $a$ and $b$.

\section{MIMO Channel Model}

2.1. General Description. A flat fading MIMO system equipped with $N$ transmit and $M$ receive antennas is showed in Figure 1. The transmit and receive signals are expressed by vectorized forms, $\mathbf{X}=\left[x_{1}, x_{2}, \ldots, x_{N}\right]^{T}$ and $\mathbf{S}=$ $\left[s_{1}, s_{2}, \ldots, s_{M}\right]^{T}$. The input-output relationship is given by

$$
\mathbf{S}=\mathbf{H X}+\mathbf{N}
$$

where $\mathbf{N}$ corresponds to the additive noise matrix, $\mathbf{H}$ is the time-variate channel fading matrix which can be expressed as

$$
\mathbf{H}(t)=\left[\begin{array}{cccc}
h_{1,1}(t) & h_{1,2}(t) & \cdots & h_{1, N}(t) \\
h_{2,1}(t) & h_{2,2}(t) & \cdots & h_{2, N}(t) \\
\vdots & \vdots & \ddots & \vdots \\
h_{M, 1}(t) & h_{M, 2}(t) & \cdots & h_{M, N}(t)
\end{array}\right],
$$

where $h_{i, j}(t)$ is the complex fading coefficient from $j$ th transmit antenna to $i$ th receive antenna. For simplicity, $\mathbf{H}(t)$ is transformed to a column vector containing $M N$ elements,

$$
\begin{array}{r}
\operatorname{vec}(\mathbf{H})=\left[h_{1,1}, h_{2,1}, \ldots, h_{M, 1}, h_{1,2}, h_{2,2}, \ldots,\right. \\
\left.h_{M, 2}, \ldots, h_{1, N}, h_{2, N}, \ldots, h_{M, N}\right]^{T},
\end{array}
$$

or

$$
\operatorname{vec}(\mathbf{H})=\left[h_{1}(t), h_{2}(t), \ldots, h_{M N}(t)\right]^{T} .
$$

The envelope of $h_{k}(t)$ follows Nakagami distribution, and the phase is uniformly distributed in $[0,2 \pi)$. The TCC of each subchannel is denoted by $\rho_{k}(\tau)$, and the SCC matrix of $\operatorname{vec}(\mathbf{H})$ can be described by a $M N \times M N$ matrix $\boldsymbol{\rho}_{\mathrm{R}}$.
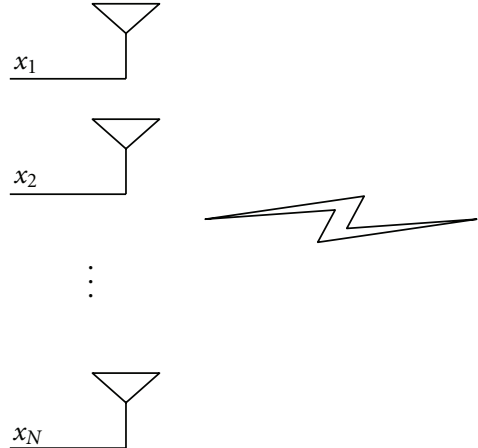
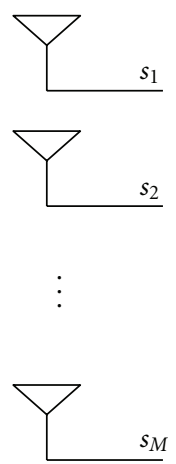

Figure 1: MIMO system with $N$ transmit and $M$ receive antennas.

2.2. Nakagami, Rayleigh, and Gamma Distribution. The probability density function (PDF) of Nakagami distribution was given in [2] as

$$
f_{R}(r)=\frac{2}{\Gamma(m)}\left(\frac{m}{\Omega}\right)^{m} r^{2 m-1} e^{-(m / \Omega) r^{2}}, \quad r \geq 0,
$$

where $\Omega=E\left[r^{2}\right]$ is the mean power, $\Gamma(\cdot)$ denotes the gamma function, and the fading parameter $m$ controls the fading degree of the propagation field.

Following [2], the squared Nakagami RV obeys a Gamma distribution and can be constructed from the sum of independent and identically distributed (i.i.d) squared Rayleigh RVs,

$$
\begin{gathered}
R=\sqrt{Y_{1}^{2}+Y_{2}^{2}+\cdots+Y_{m}^{2}}, \\
R=\sqrt{\gamma},
\end{gathered}
$$

where $Y_{i}$ and $\gamma$ denote Rayleigh and Gamma RV, respectively, whose PDFs are defined as

$$
\begin{aligned}
& f_{Y}(y)=\frac{y}{\sigma^{2}} \exp \left(-\frac{\mathrm{y}^{2}}{2 \sigma^{2}}\right), \\
& f_{\gamma}(\gamma)=\frac{1}{\Gamma(a) b^{a}} \gamma^{a-1} \mathrm{e}^{-\gamma / b},
\end{aligned}
$$

where $\sigma^{2}=\Omega / 2 m, a=m, b=\Omega / m$.

\section{Generation of Correlated Nakagami MIMO Channel}

In general, the correlated Nakagami MIMO channel simulation is to generate $M N$ Nakagami fading channels which are temporal and spatial correlated. It is assumed that the fading parameters and correlation properties are arbitrary and different.

3.1. Generating Independent Nakagami Fading. The rejection/acceptance technique is a universal generation method for nonuniform RVs, which is summarized as follows [14]

(1) Find a hat function $p(x)$ which satisfies the inequality

$$
C p(x) \geq q(x)
$$




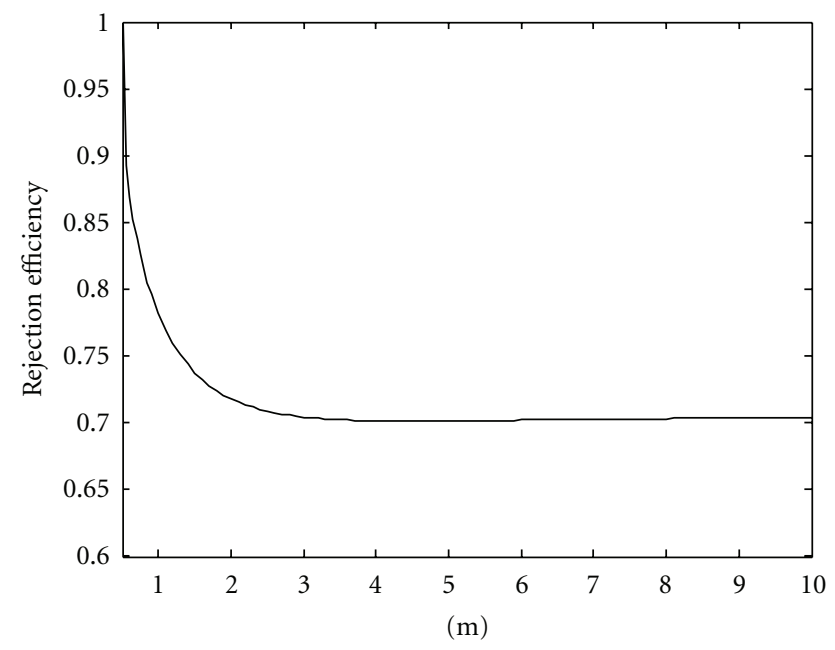

FIGURE 2: Rejection efficiency of the new method.

where $q(x)$ is the required distribution and $C$ is the rejection constant.

(2) Generate a RV $X$ with PDF $p(x)$ and a RV G uniformly distributed in $[0, C p(X)]$.

(3) If $G \leq q(X), X$ is accepted. Otherwise, it is rejected.

The main difficulty of this technique lies in finding a hat function which is close to the required distribution and satisfies the inequality. In this paper, we adopt a new truncated Gaussian distribution function as the hat function for arbitrary $m$. Using (8) and strict mathematical derivation, the hat function and rejection constant are founded as

$$
\begin{aligned}
p(x) & =\frac{(1 / \sqrt{2 \pi} \sigma) e^{-\left(x-x_{0}\right)^{2} / 2 \sigma^{2}}}{1 / 2+(1 / 2) \operatorname{erf}\left(x_{0} / \sqrt{2} \sigma\right)}, \quad x \geq 0, \\
c & =b \sqrt{2 \pi} \sigma\left(\frac{1}{2}+\frac{1}{2} \operatorname{erf}\left(\frac{x_{0}}{\sqrt{2} \sigma}\right)\right),
\end{aligned}
$$

where $x_{0}=\sqrt{(2 m-1) \Omega / 2 m}, \sigma^{2}=\Omega / 2 m$, and

$$
b=\frac{2^{3 / 2-m}(m / \Omega)^{m}(\Omega(2 m-1) / m)^{m-1 / 2} e^{1 / 2-m}}{\Gamma(m)} .
$$

An important measure for the quality of the rejection algorithm is the acceptance probability or rejection efficiency which involves the closeness between the Nakagami PDF and the hat function. Figure 2 plots the rejection efficiency curve of our proposed method. It shows that the efficiency decreases for high values of $m$ but better than $70 \%$. However, the rejection efficiency of [8] is only $65.75 \%$ and $66.67 \%$ in cases of $0.5 \leq m \leq 1$ and $m \geqslant 1$, while the method in [9] becomes inefficient as $m$ increases and the efficiency is below $64 \%$ mostly.

3.2. Introducing Temporal Correlation. For stationary random processes, the TCC of the samples is well approximate with the TCC of their statistical ranks [15]. Reference [11, Equation (6)] gives the assumption without proving that

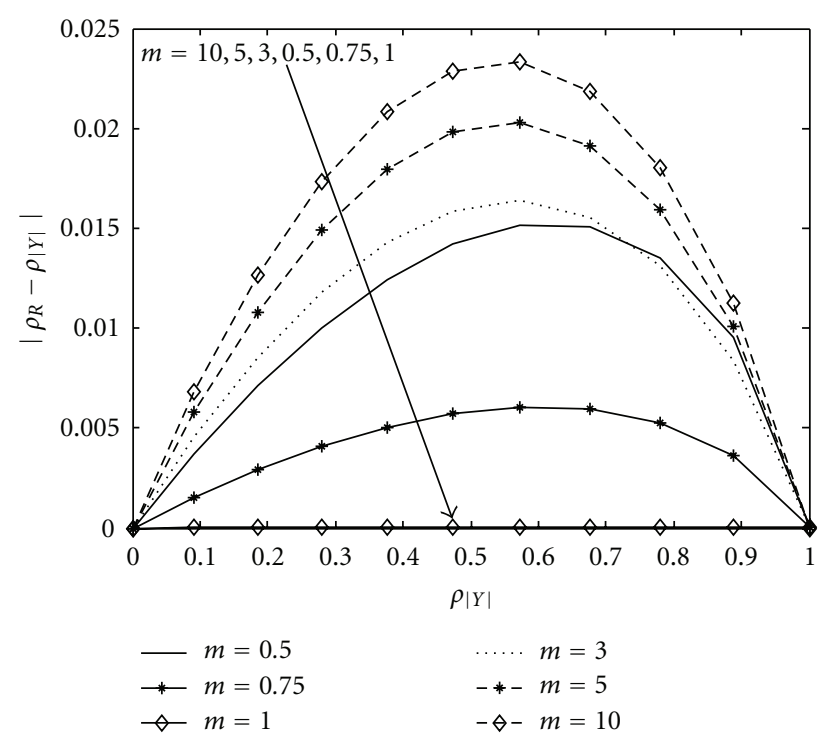

FIGURE 3: The absolute error between $\rho_{R}(\tau)$ and $\rho_{|Y|}(\tau)$.

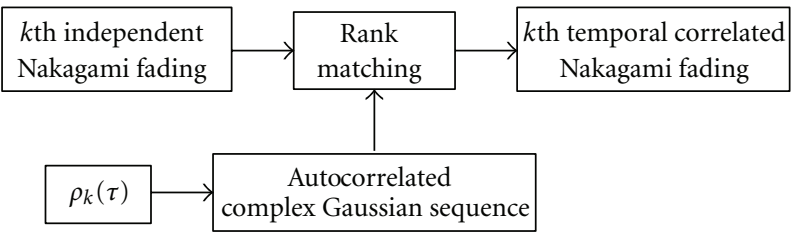

Figure 4: Introducing temporal correlation.

the TCC of Nakagami envelope approximately equal to the corresponding Rayleigh envelope $\rho_{R}(\tau) \approx \rho_{|Y|}(\tau)$ which means the TCCs of their statistical ranks are almost same.

The Nakagami envelope autocorrelation function is given by [2]:

$$
f_{R}(\tau)=\frac{\Omega \Gamma^{2}(m+0.5)}{m \Gamma^{2}(m)} F_{1}\left(-0.5,-0.5 ; m ; \rho_{Y^{2}}(\tau)\right),
$$

where $\rho_{Y^{2}}(\tau)$ is the autocorrelation coefficient of squared Rayleigh envelope and $F_{1}(a, b ; c ; d)$ is the hypergeometric function. The statistical properties of Nakagami sequence can be found as

$$
\begin{gathered}
E[R]=\frac{\Gamma(m+0.5)}{\Gamma(m)}\left(\frac{\Omega}{m}\right)^{1 / 2}, \\
\operatorname{var}(R)=\Omega-\frac{\Omega \Gamma^{2}(m+0.5)}{m \Gamma^{2}(m)} .
\end{gathered}
$$

According to the definition of autocorrelation coefficient, we obtain

$$
\rho_{R}(\tau)=\frac{1-F_{1}\left(-0.5,-0.5 ; m ; \rho_{Y^{2}}(\tau)\right)}{1-\left(m \Gamma^{2}(m) / \Gamma^{2}(m+0.5)\right)} .
$$

Following [16], we have

$$
\rho_{|Y|}(\tau)=\frac{1-F_{1}\left(-0.5,-0.5 ; 1 ; \rho_{Y^{2}}(\tau)\right)}{1-4 / \pi},
$$




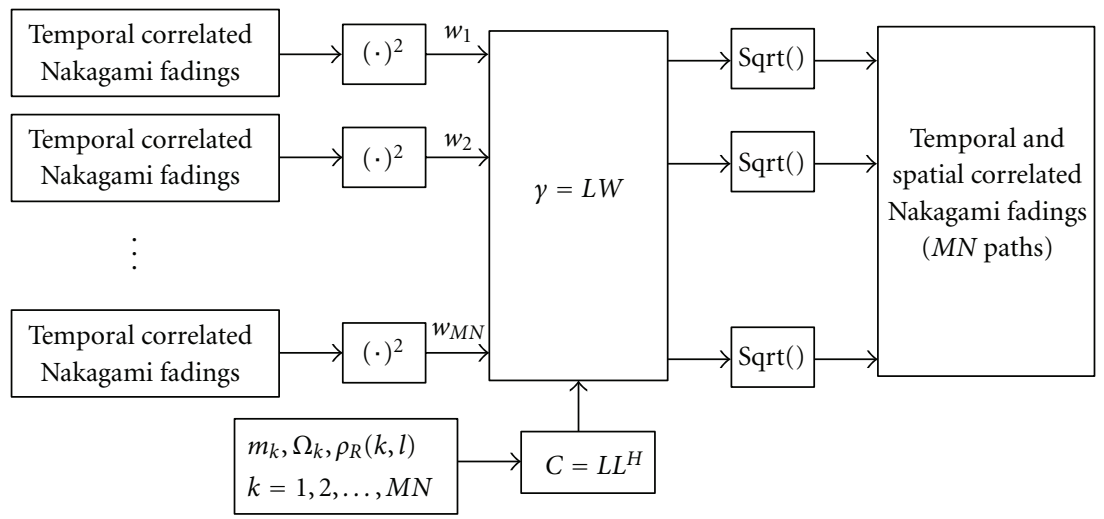

FIGURE 5: Introducing spatial correlation.

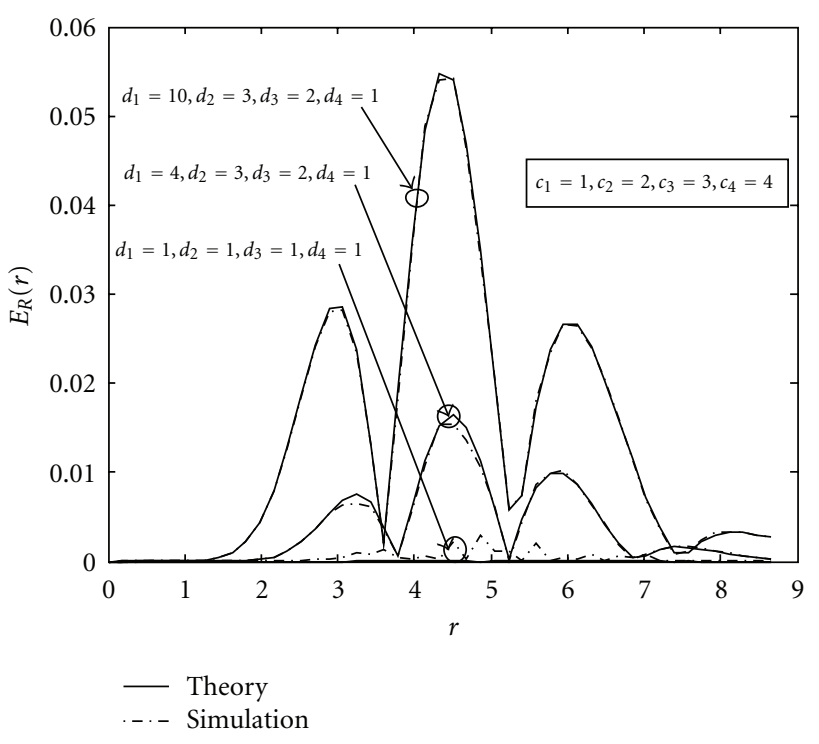

FIGURE 6: Comparison of the absolute error of Nakagami PDF.

where $\rho_{|Y|}(\tau)$ is the autocorrelation coefficient of Rayleigh envelope.

It is difficult to get the accurate relationship expression between $\rho_{R}(\tau)$ and $\rho_{|Y|}(\tau)$. A numerical method is used to investigate the absolute error for different $m$. The numerical results are plotted in Figure 3, which shows the maximum difference between two TCC curves is less than 0.025 which is fairly small for most practical purposes.

Thus, we can rearrange the Nakagami sequence to match the statistical rank of the Rayleigh sequence in order to introduce the desired temporal correlation (see Figure 4). Moreover, there are many efficient methods to generate the reference Rayleigh sequence (or complex Gaussian sequence) with desired autocorrelation, such as the AR model [17] and the MEPA method [18]. It should be emphasized that the temporal correlated complex Gaussian sequence here can be transformed to the truncated Gaussian sequence for rejecting in (9).

3.3. Introducing Spatial Correlation. We introduce the spatial correlation for $M N$ Nakagami fading subchannels following the flowchart in Figure 5. The key step of this procedure is generating $M N$ correlated Gamma RVs whose square root yields the desired correlated Nakagami MIMO channel. There are two commonly used methods, the decomposition method [12] and the Simon method [19]. Although the Simon method shows better accuracy, it is more complex and has some constraints on the prespecified statistical parameters. The decomposition method is more versatile and simpler, which can be expressed as

$$
\gamma=\mathbf{L w}
$$

where $\mathbf{w}=\left[w_{1} \cdots w_{M N}\right]^{\mathrm{T}}$ is the normalized Gamma RV vector, $w_{i} \sim \Upsilon\left(a_{i}, b_{i}\right)$ and $a_{i} b_{i}^{2}=1, y=\left[\gamma_{1} \cdots \gamma_{M N}\right]^{\mathrm{T}}$ is the desired cross-correlated Gamma RV vector, and $\mathbf{L}$ denotes a low triangular matrix, which is the Cholesky decomposition on the covariance matrix of $\gamma$,

$$
\mathbf{C}_{\gamma}=\mathbf{L L}^{\mathrm{H}} .
$$

It can be derived that $\mathbf{C}_{\gamma}, a_{i}, b_{i}$ are determined by the specified simulation parameters,

$$
\begin{gathered}
c_{\gamma}(k, l) \approx \rho_{R}(k, l) \sqrt{\frac{\left(1+1 / m_{k}\right)^{2} \Omega_{k}^{4}}{m_{k}} \frac{\left(1+1 / m_{l}\right)^{2} \Omega_{l}^{4}}{m_{l}}}, \\
a_{i}=\frac{1}{b_{i}^{2}}, \quad i=1 \sim M N, \\
b_{i}=\frac{l_{i, i}}{\sqrt{m_{i} \sum_{j=1}^{i} l_{i, j}^{2}-\sum_{j=1}^{i-1} l_{i, j} / b_{j}}} .
\end{gathered}
$$

The decomposition method assumes that the linear summation of independent Gamma RVs follows Gamma distribution. In the following, we will analyze the simulation error caused by this assumption. Firstly, the $i$ th crosscorrelated Gamma RV $\gamma_{i}$ of (15) can be written as

$$
\begin{aligned}
\gamma_{i} & =\sum_{k=1}^{i} l_{i, k} w_{k}=l_{i, 1} w_{1}+l_{i, 2} w_{2}+\cdots+l_{i, i} w_{i} \\
& =w_{1}^{\prime}+w_{2}^{\prime}+\cdots+w_{i}^{\prime},
\end{aligned}
$$




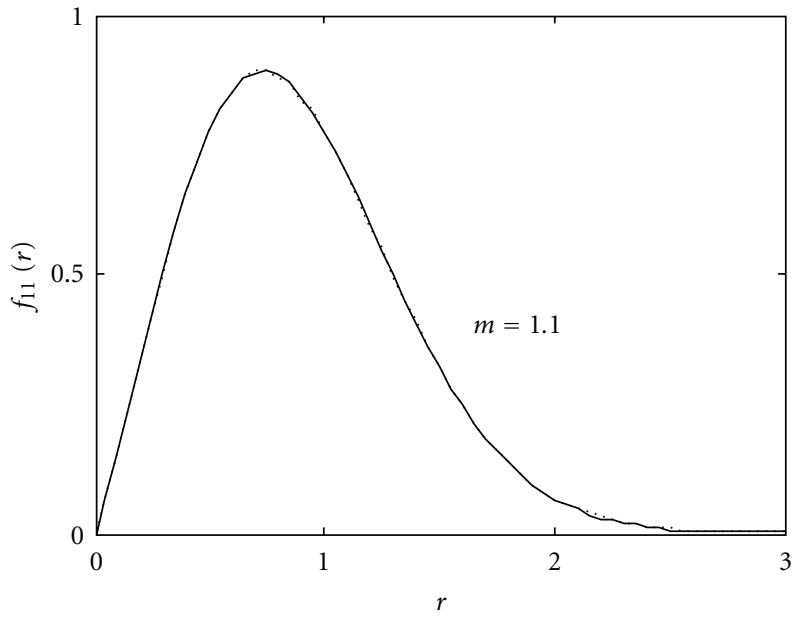

(a)

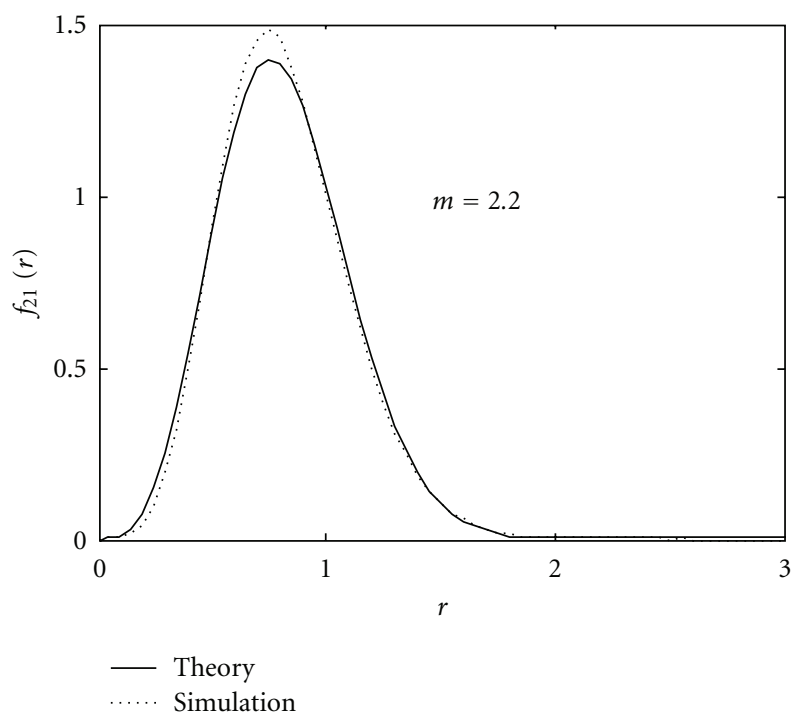

(c)

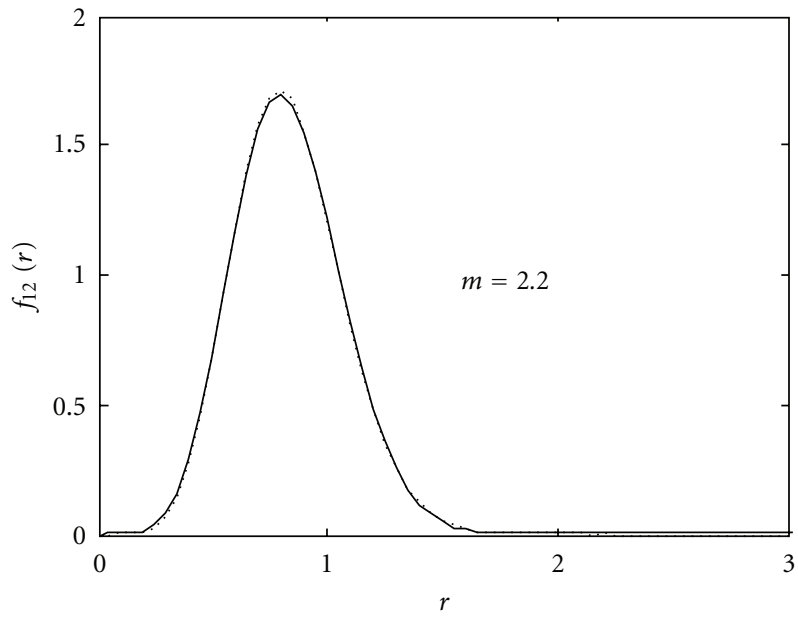

(b)

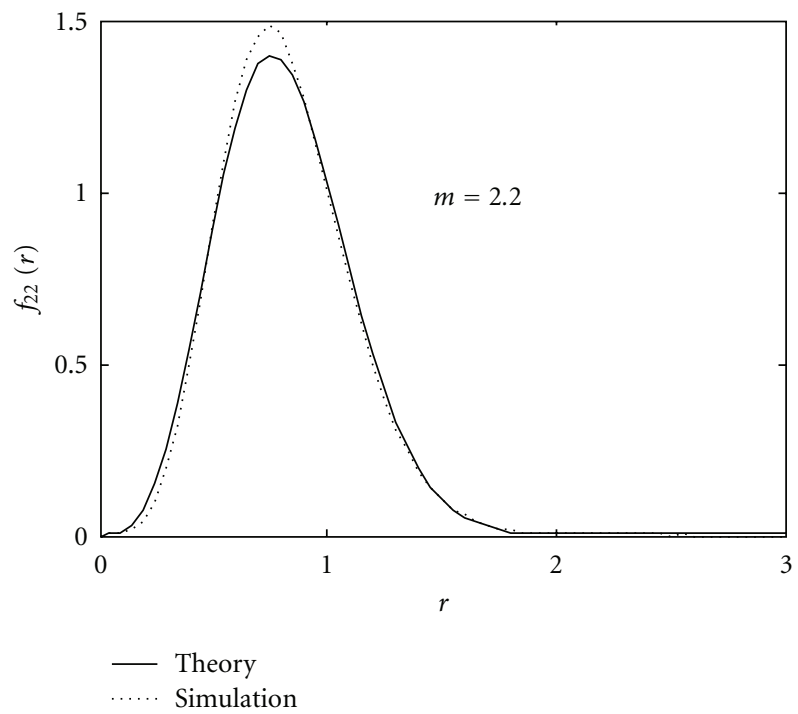

(d)

Figure 7: The PDFs of simulated subchannel fading envelope.

where $w_{i}^{\prime}=l_{i} w_{i}$ denotes a new independent Gamma RV which follows $w_{i}^{\prime} \sim \Upsilon\left(c_{i}, d_{i}\right)$, and the new distribution parameters yield

$$
c_{i}=a_{i}, \quad d_{i}=l_{i} b_{i} .
$$

The exact PDF of $y_{i}$ can be found as [20, Equation (2.9)]:

$$
f_{\gamma}(z)=\frac{A \sum_{K=0}^{\infty} \delta_{k} z^{\rho+k-1} e^{-z / d_{1}}}{\left(\Gamma(\rho+k) d_{1}^{\rho+k}\right)}
$$

where $d_{1}=\min \left\{d_{i}\right\}, A=\prod_{i=1}^{n}\left(d_{1} / d_{i}\right)^{c_{i}}, \rho=\sum_{i=1}^{n} c_{i}>0$, and

$$
\delta_{k+1}=\frac{1}{k+1} \sum_{i=1}^{k+1} i B_{k} \delta_{k+1-i}, \quad \delta_{0}=1,
$$

where $B_{k}=\sum_{i=1}^{n} c_{i}\left(1-d_{1} / d_{i}\right)^{k} / k$.
The straightforward transformation $R=\sqrt{\gamma}$ leads the simulation PDF of Nakagami RV as

$$
f_{R}(r)=2 r f_{\gamma}\left(r^{2}\right)
$$

Finally, the theoretical expression for absolute error of simulated Nakagami PDF is obtained as

$$
E_{R}(r)=\left|2 r f_{R}\left(r^{2}\right)-\frac{2}{\Gamma(m)}\left(\frac{m}{\Omega}\right)^{m} r^{2 m-1} e^{-(m / \Omega) r^{2}}\right| .
$$

Figure 6 compares the simulated results of Nakagami PDF error with the values calculated from the exact expression (23). In the simulation, $10^{6}$ samples of the Nakagami RV are generated by four independent Gamma RVs with different parameters sets of $c_{i}$ and $d_{i}$. It can be seen that a very good agreement between the simulated results and the exact expression is obtained. Anyway, the absolute error of Nakagami PDF is very small which means the decomposition method can achieve satisfactory performance. 


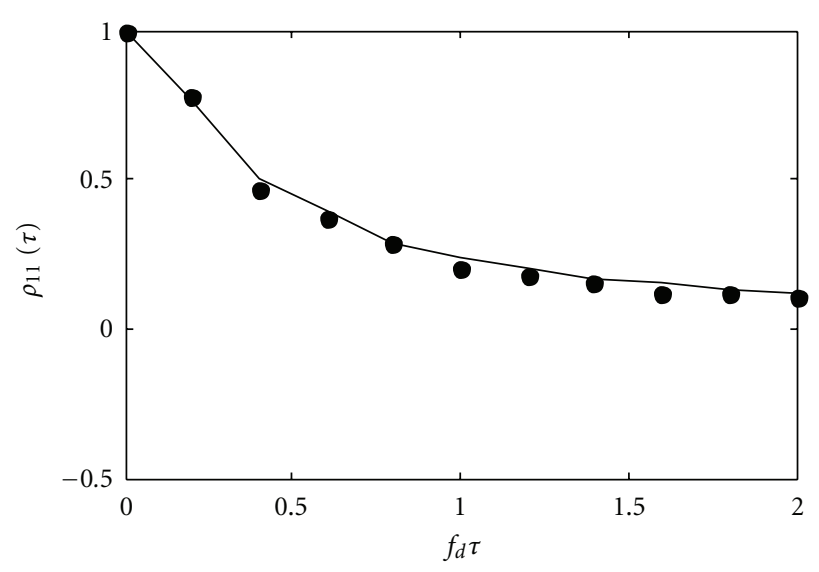

(a)

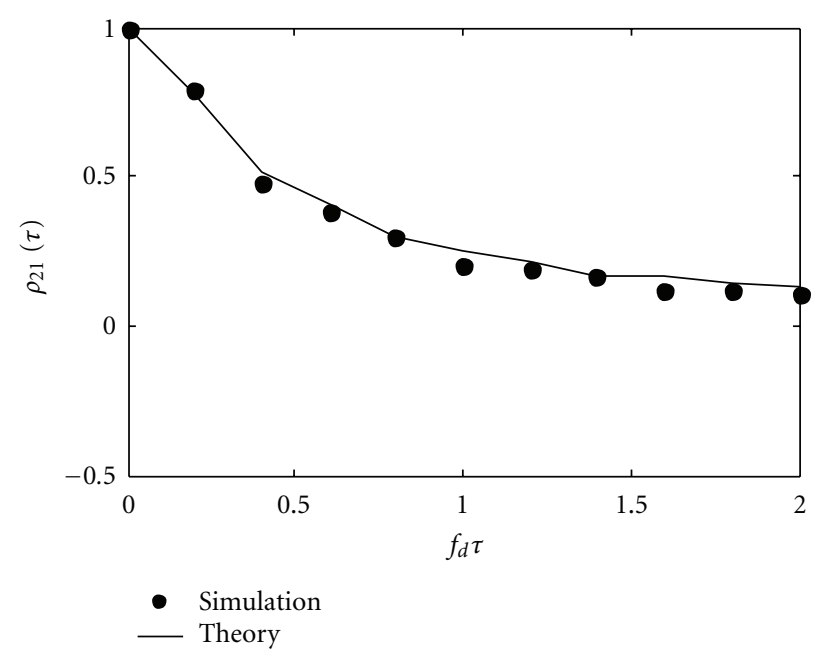

(c)

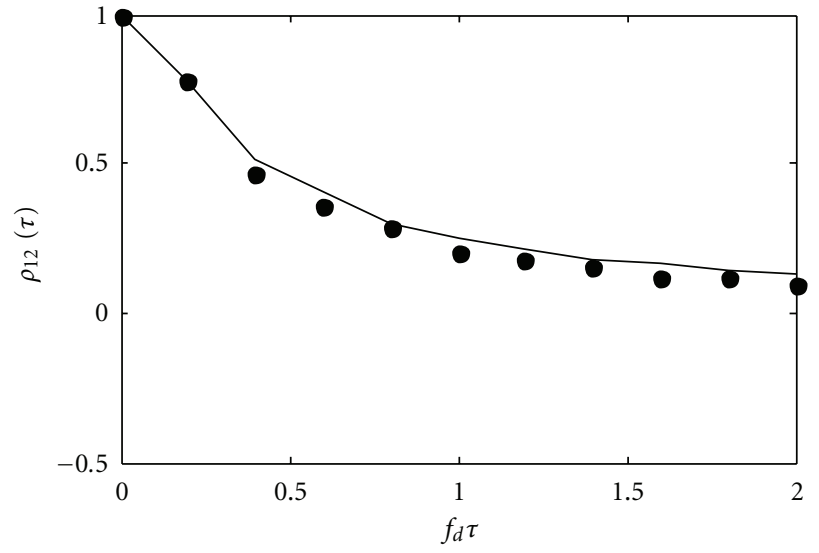

(b)

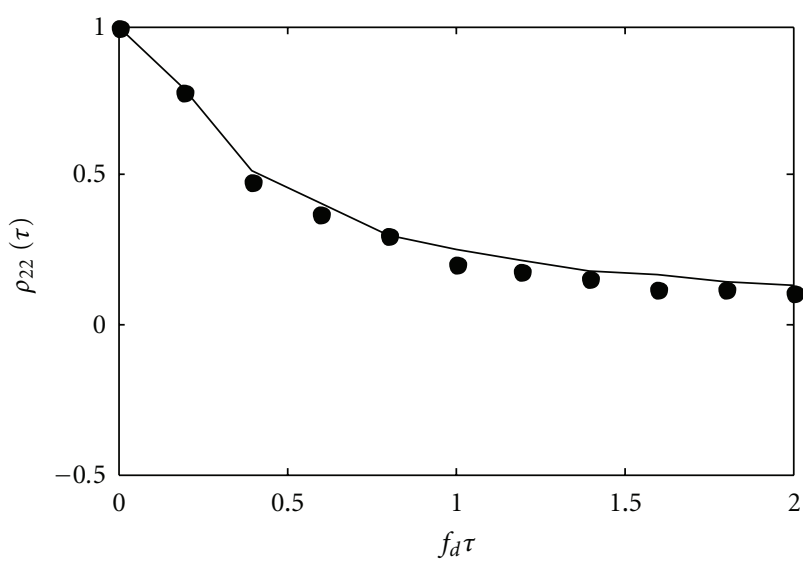

- Simulation

- Theory

FIgURE 8: The TCCs of simulated subchannel fading envelope.

\section{Simulation Results}

To illustrate the behavior of the proposed channel simulator, we consider a MIMO system with $N=2$ at the mobile station (MS), $M=2$ at the base station (BS). The fading characteristics of four subchannels are different:

$$
\mathbf{m}=\left[\begin{array}{ll}
1.1 & 3.3 \\
2.2 & 4.4
\end{array}\right], \quad \boldsymbol{\Omega}=\left[\begin{array}{cc}
1 & 0.75 \\
0.75 & 1
\end{array}\right]
$$

The MS is assumed to be surrounded by several scatterers which results in a low correlation between two antennas

$$
\boldsymbol{\rho}_{\mathrm{MS}}=\left[\begin{array}{cc}
1 & 0.3 \\
0.3 & 1
\end{array}\right]
$$

while the antennas of BS set high and have no local scatters,

$$
\boldsymbol{\rho}_{\mathrm{BS}}=\left[\begin{array}{cc}
1 & 0.91 \\
0.91 & 1
\end{array}\right] \text {. }
$$

Hence, the SCC matrix is given by [21]:

$$
\rho_{\mathrm{R}}=\boldsymbol{\rho}_{\mathrm{MS}} \otimes \boldsymbol{\rho}_{\mathrm{BS}}=\left[\begin{array}{cccc}
1 & 0.91 & 0.3 & 0.273 \\
0.91 & 1 & 0.273 & 0.3 \\
0.3 & 0.273 & 1 & 0.91 \\
0.273 & 0.3 & 0.91 & 1
\end{array}\right],
$$

where $\otimes$ denotes Kronecker product.

A plausible model for the angle of arrival (AOA) of the receiving signals is Von Mise distribution, which is defined as [22]:

$$
p(\alpha)=\frac{\exp \left\{k \cos \left(\alpha-\alpha_{0}\right)\right\}}{2 \pi I_{0}(k)}, \quad \alpha \in(-\pi, \pi] .
$$

Under this scatting environment, the theoretical expression of TCC is given by [22] as

$$
\rho_{Y^{2}}(\tau)=\left|\frac{I_{0}\left(\sqrt{k^{2}-\left(2 \pi f_{d} \tau\right)^{2}+j 4 \pi k f_{d} \cos \left(\alpha_{0}\right) \tau}\right)}{I_{0}(k)}\right|^{2},
$$


where $I_{0}(\cdot)$ is the zero-order modification Bessel function, $\alpha_{0}$ denotes the mean direction of AOA, $k \geq 0$ controls the angular spread, and $f_{d}$ is the maximum Doppler frequency. Antenna arrays at both ends are assumed to be small spacing (less than half wavelength), so the temporal correlation properties of receiving signals on each receiving antenna are almost same.

In the simulation, $10^{6}$ samples of each subchannel are generated based on the proposed scheme. Figure 7 shows that the simulated PDFs of fading envelope match the theoretical curves very well under four fading scenarios. The corresponding statistical parameters are estimated by maximum likelihood method as

$$
\hat{\mathbf{m}}=\left[\begin{array}{ll}
1.0982 & 3.3073 \\
2.1983 & 4.4144
\end{array}\right], \quad \hat{\mathbf{\Omega}}=\left[\begin{array}{ll}
1.0010 & 0.7506 \\
0.7506 & 1.0006
\end{array}\right],
$$

which is also very close to the desired ones.

We next look at the spatial and temporal correlation coefficients of the above outputs. In the simulation, we assume that four subchannels experience almost the same no-isotropic $(k=5)$ scattering scenarios for small antenna spacing. The TCC of each output channel is showed in Figure 8 , where $f_{d} \tau$ means the normalized time delay. It is showed that the simulation curves agree well with the desired ones. The SCC matrix is calculated by output samples ending up with

$$
\hat{\rho}_{R}=\left[\begin{array}{cccc}
1 & 0.8778 & 0.3074 & 0.2765 \\
0.8778 & 1 & 0.2771 & 0.3110 \\
0.3074 & 0.2771 & 1 & 0.9076 \\
0.2765 & 0.3110 & 0.9076 & 1
\end{array}\right] .
$$

Comparing to (27), it is found that the relative errors are $3.54 \%, 2.47 \%$, and $1.27 \%$, respectively.

\section{Conclusions}

In this paper, a new framework for simulating the spatial and temporal correlated MIMO Nakagami fading channels was proposed. It can be applied on arbitrary fading parameters and prespecified spatial-temporal correlation coefficients with a satisfactory accuracy. Moreover, the new simulation method is low complexity and applicable for large-scale and real-time channel simulation scenarios. The most complex part of this simulator is to generate $M N$ complex Gaussian sequences which can be accomplished by well-established methods.

\section{Acknowledgments}

This work is supported by National Nature Science Foundation of China (no. 61102068), the Aviation Science Foundation of China (no. 2009ZC52036), Nanjing University of Aeronautics and Astronautics Research Funding (no. NP2011036 and NS2011013), and China Postdoctoral Science Foundation funded project (no. 20110490389).

\section{References}

[1] E. Telatar, "Capacity of multi-antenna Gaussian channels," European Transactions on Telecommunications, vol. 10, no. 6, pp. 585-595, 1999.

[2] M. Nakagami, The m-Distribution: A General Formula of Intensity Distribution of Rapid Fading Statistical Methods of Radio Wave Propagation, W.C. Hoffman, Japan, 1960.

[3] M. Matthaiou, D. I. Laurenson, and J. S. Thompson, "A MIMO channel model based on the Nakagami-faded spatial eigenmodes," IEEE Transactions on Antennas and Propagation, vol. 56, no. 5, pp. 1494-1497, 2008.

[4] C. Zhong, K. K. Wong, and S. Jin, "Capacity bounds for MIMO Nakagami-m fading channels," IEEE Transactions on Signal Processing, vol. 57, no. 9, pp. 3613-3623, 2009.

[5] K. W. Yip and T. S. Ng, "A simulation model for nakagami$\mathrm{m}$ fading channels, $\mathrm{m}<1$," IEEE Transactions on Communications, vol. 48, no. 2, pp. 214-221, 2000.

[6] Q. T. Zhang, "Decomposition technique for efficient generation of correlated Nakagami fading channels," IEEE Journal on Selected Areas in Communications, vol. 18, no. 11, pp. 23852392, 2000.

[7] N. C. Beaulieu and C. Cheng, "Efficient nakagami- $\mathcal{M}$ fading channel simulation," IEEE Transactions on Vehicular Technology, vol. 54, no. 2, pp. 413-424, 2005.

[8] L. Cao and N. C. Beaulieu, "Simple efficient methods for generating independent and bivariate Nakagami-m fading envelope samples," IEEE Transactions on Vehicular Technology, vol. 56, no. 4 I, pp. 1573-1579, 2007.

[9] M. Matthaiou and D. I. Laurenson, "Rejection method for generating Nakagami-m independent deviates," Electronics Letters, vol. 43, no. 25, pp. 1474-1475, 2007.

[10] Y. Ma and D. Zhang, "A method for simulating complex nakagami fading time series with nonuniform phase and prescribed autocorrelation characteristics," IEEE Transactions on Vehicular Technology, vol. 59, no. 1, Article ID 5223623, pp. 29-35, 2010.

[11] J. C. S. Silveira Santos Filho and M. D. Yacoub, "On the simulation and correlation properties of phase-envelope nakagami fading processes," IEEE Transactions on Communications, vol. 57, no. 4, pp. 906-909, 2009.

[12] K. Zhang, Z. Song, and Y. L. Guan, "Simulation of nakagami fading channels with arbitrary cross-correlation and fading parameters," IEEE Transactions on Wireless Communications, vol. 3, no. 5, pp. 1463-1468, 2004.

[13] L. C. Tran, T. A. Wysocki, and J. Seberry, "A generalized algorithm for the generation of correlated Rayleigh fading envelopes in radio channels," in Proceedings of the 19th IEEE International Parallel and Distributed Processing Symposium, (IPDPS'05), vol. 13, p. 238, Denver, Colo, USA, 2005.

[14] A. Papoulis and S. U. Pillai, Probability Random Variables and Stochastic Processes, McGraw-Hill, Boston, Mass, USA, 4th edition, 2002.

[15] E. L. Lehmann and H. L. M. D'Abrera, Nonparametrics: Statistical Methods Based on Ranks, McGraw-Hill, New York, NY, USA, 1975.

[16] W. C. Jakes, Microwave Mobile Communications, Wiley, New York, NY, USA, 1974

[17] K. E. Baddour and N. C. Beaulieu, "Autoregressive modeling for fading channel simulation," IEEE Transactions on Wireless Communications, vol. 4, no. 4, pp. 1650-1662, 2005. 
[18] Q. M. Zhu, D. Z. Xu, and X. M. Chen, "Modified channel model for non-isotropic scattering environments," in Proceedings of the International Conference on Wireless Communications \& Signal Processing, (WCSP '09), pp. 1-5, Nanjing, China, November 2009.

[19] C. H. Simon, "Generation of Poisson and Gamma random vectors with given marginal and covariance matrix," Journal of Statistical Computation and Simulation, vol. 47, no. 1, pp. $1-10,1993$.

[20] P. G. Moschopoulos, "The distribution of the sum of independent gamma random variables," Annals of the Institute of Statistical Mathematics, vol. 37, no. 1, pp. 541-544, 1985.

[21] J. P. Kermoal, L. Schumacher, K. I. Pedersen, P. E. Mogensen, and F. Frederiksen, "A stochastic MIMO radio channel model with experimental validation," IEEE Journal on Selected Areas in Communications, vol. 20, no. 6, pp. 1211-1226, 2002.

[22] A. Abdi, J. A. Barger, and M. Kaveh, "A parametric model for the distribution of the angle of arrival and the associated correlation function and power spectrum at the mobile station," IEEE Transactions on Vehicular Technology, vol. 51, no. 3, pp. 425-434, 2002. 

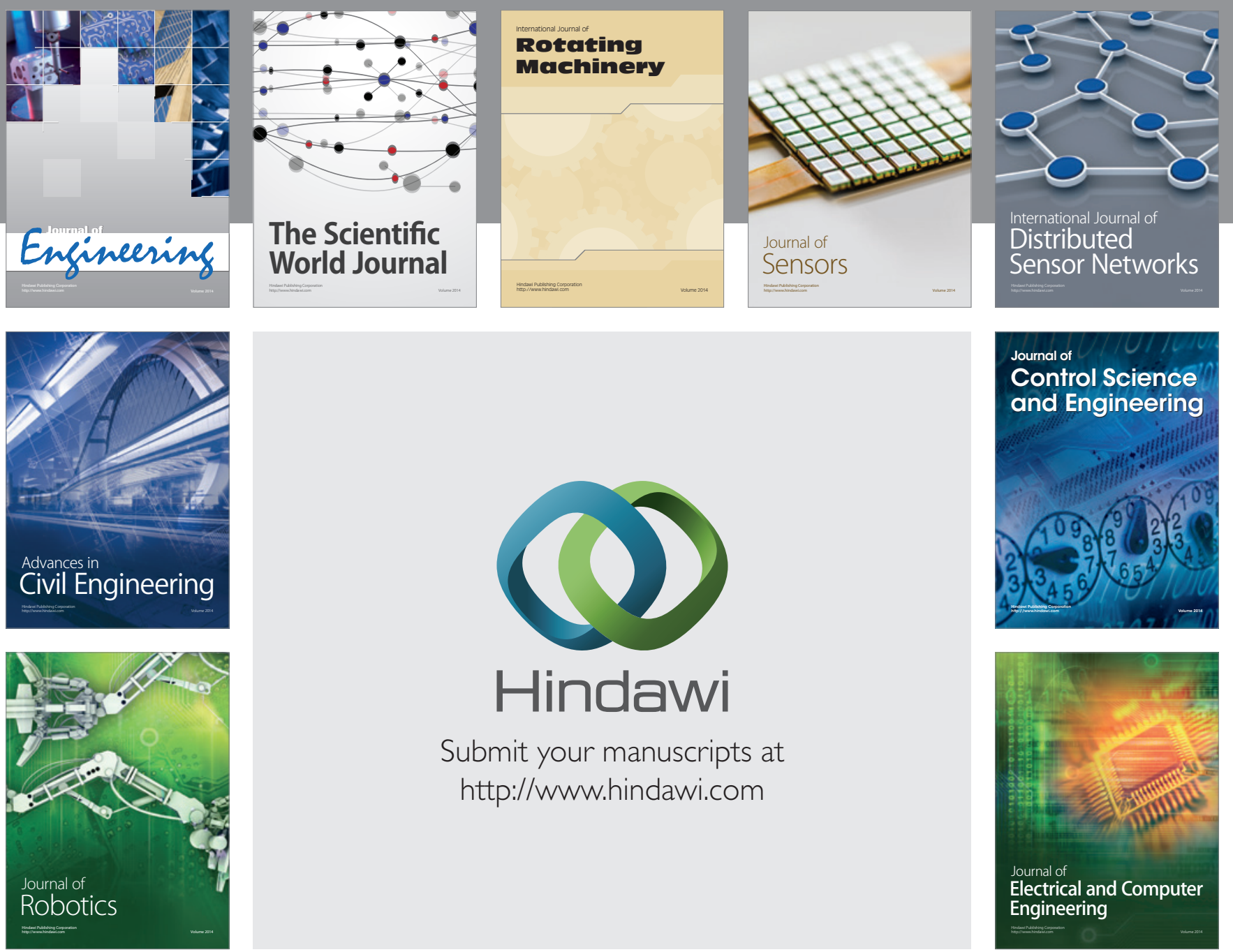

Submit your manuscripts at

http://www.hindawi.com
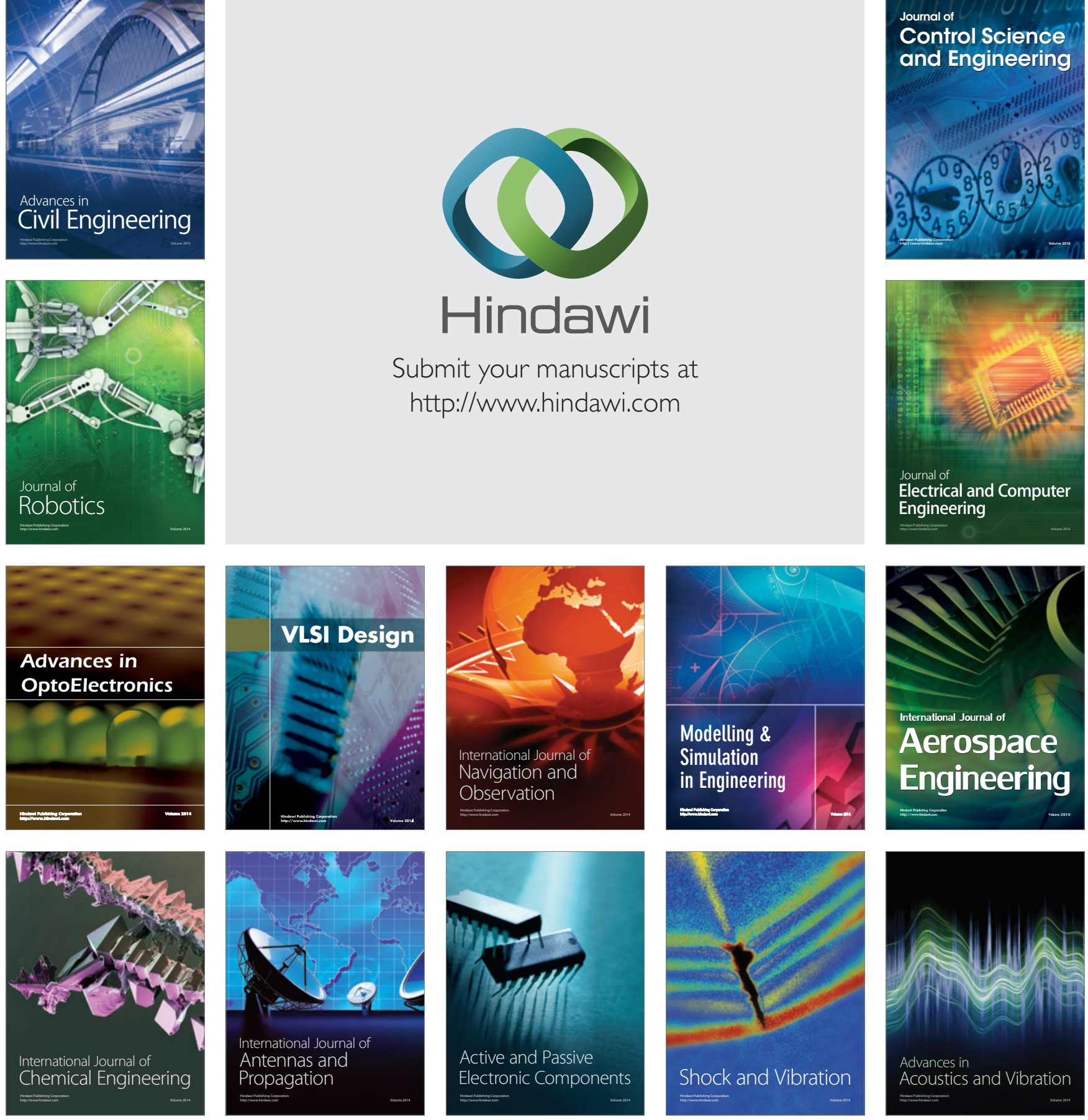